\title{
A Novel OFDMA Ranging Method Exploiting Multiuser Diversity
}

\author{
Jianqiang Zeng and Hlaing Minn (Contact Author) \\ Department of Electrical Engineering, University of Texas at Dallas \\ Email: $\{$ jxz051000, hlaing.minn $\} @$ utdallas.edu
}

\begin{abstract}
This paper addresses initial ranging (uplink synchronization and power control) for TDD OFDMA systems. Exploiting the channel knowledge from the downlink control channel together with initial power control, we develop a novel initial ranging method which provides multiuser diversity gain and significant power saving for the subscriber stations. We present a new ranging signal design which provides multiuser diversity gain and facilitates new efficient low-complexity algorithms for multiuser ranging signal detection, timing estimation and power estimation. The advantages of the proposed approach over existing methods in terms of the ranging signal detection performance, the number of ranging frames required to finish the ranging process, the timing and power estimation performance, the ranging transmission power saving at the subscriber stations, and the complexity saving at the base station are illustrated by analytical and simulation results.
\end{abstract}

\section{INTRODUCTION}

In OFDMA systems [1] [2], uplink synchronization is accomplished by initial ranging process. The existing ranging methods [3]-[6] have a common disadvantage of high complexity. In this paper we present a novel initial ranging method for time-division duplexing (TDD) OFDMA systems which provides multiuser diversity gains while requiring only lowcomplexity algorithms for multiuser ranging signal detection, timing offset estimation and power estimation.

\section{System Description And Signal Model}

Consider an uplink OFDMA system with $N_{r}$ RSSs, $N_{d}$ data subscriber stations (DSSs) and $N$ subcarriers. After excluding DC and null subcarriers, the remaining subcarriers are grouped into $Q_{r}$ ranging subchannels and $Q_{d}$ data subchannels. The numbers of left and right null subcarriers are denoted by $\gamma_{L N}$ and $\gamma_{R N}$, respectively. Each ranging subchannel has $\gamma_{r}$ subcarriers and each data subchannel has $\gamma_{d}$ subcarriers. The indices of subcarriers corresponding to the $i$ th RSS and the $j$ th DSS are denoted as $\mathcal{J}_{i, r}$ and $\mathcal{J}_{j, d}$, respectively.

In the subcarrier domain at the $m$ th OFDM symbol interval, the length- $N$ ranging code vector for the $i$ th RSS and the length- $N$ data vector for the $j$ th DSS are denoted by $\mathbf{X}_{i, r}^{(m)}$ and $\mathbf{X}_{j, d}^{(m)}$. The corresponding $n$th elements $(n \in\{0, \ldots, N-1\})$ are given by ${ }^{1}$

$$
\mathrm{X}_{i, \star}^{(m)}(n)= \begin{cases}A_{i, \star} C_{i, \star}^{(m)}(l), & n \in \mathcal{J}_{i, \star}(l) \\ 0, & l=0, \ldots, \gamma_{\star}-1 \\ 0, & \text { otherwise }\end{cases}
$$

\footnotetext{
${ }^{1}$ In the rest of the paper, the subscript $\star$ denotes whether $r$ or $d$ representing ranging or data.
}

where $C_{i, r}^{(m)}(l)$ and $C_{j, d}^{(m)}(l)$ are ranging and data symbols, respectively, with $\left|C_{i, r}^{(m)}(l)\right|=E\left[\left|C_{j, d}^{(m)}(l)\right|^{2}\right]=1$, and $\left\{A_{i, r}, A_{j, d}>0\right\}$ are amplitude scaling factors. Denote the $N$-point inverse discrete Fourier transform $\left(\operatorname{IDFT}_{N}\right)$ of $\mathbf{X}_{i, r}^{(m)}$ and $\mathbf{X}_{j, d}^{(m)}$ by $\left[\mathrm{x}_{i, r}^{(m)}(0), \ldots, \mathrm{x}_{i, r}^{(m)}(N-1)\right]^{T}$ and $\left[\mathrm{x}_{i, d}^{(m)}(0), \ldots, \mathrm{x}_{i, d}^{(m)}(N-1)\right]^{T}$. Then the time domain signal samples of the $i$ th RSS and the $j$ th DSS are given by

$$
\mathrm{x}_{i, \star}(n)= \begin{cases}\mathrm{x}_{i, \star}^{(m)}\left(l-N_{g}\right) & n=m\left(N+N_{g}\right)+l, \\ & l=0, \ldots, N+N_{g}-1 \\ & m=0, \ldots, M-1 \\ 0, & \text { otherwise }\end{cases}
$$

where $M$ is the number of OFDM symbols per uplink frame.

We consider independent multi-path Rayleigh fading channels with slow fading (quasi-static over the ranging period). The sample-spaced channel impulse response taps (including the transmit and receive filters) for the $i$ th RSS and the $j$ th DSS are denoted by $\left\{h_{i, r}(l)\right\}$ and $\left\{h_{j, d}(l)\right\}, l=0, \ldots, L-1$. The channel output samples for the $i$ th $\operatorname{RSS}\left\{y_{i, r}(n)\right\}$ and for the $j$ th DSS $\left\{y_{j, d}(n)\right\}$ are given by

$$
y_{i, \star}(n)=\sum_{l=0}^{L-1} h_{i, \star}(l) x_{i, \star}\left(n-l-d_{i, \star}\right)
$$

where $d_{i, r}$ and $d_{j, d}$ are the transmission delays for the $i$ th RSS and the $j$ th DSS, respectively. The maximum possible delay $d_{\max }$ is the round-trip transmission delay for a user at the cell boundary. The CP length for an RSS should be designed such that $N_{g} \geq d_{\max }+L-1$.

Then the time domain received signal at the BS can be expressed as

$$
y(n)=\sum_{i=0}^{N_{r}-1} y_{i, r}(n)+\sum_{j=0}^{N_{d}-1} y_{j, d}(n)+\omega(n)
$$

where $\{\omega(n)\}$ are independent and identically-distributed (i.i.d.), circularly-symmetric complex Gaussian noise samples with zero mean and variance $\sigma_{\omega}^{2}$.

\section{Proposed Ranging Signal Design}

We consider an OFDMA system with $Q_{r}$ ranging subchannels, where each ranging subchannel is composed of $\gamma_{r}$ contiguous subcarriers. The ranging subchannels are spread out over the whole frequency band with approximately equal spacing. The subcarrier assignment for the $i$ th ranging subchannel $\left(i=0, \ldots, Q_{r}-1\right)$ is defined as

$$
\mathcal{J}_{i, r}=\left\{\gamma_{L N}+i \cdot \Delta+n: n=0, \ldots, \gamma_{r}-1\right\}
$$

where $\Delta=\left\lfloor\frac{N_{\text {used }}-\gamma_{r}}{Q_{r}-1}\right\rfloor$ and $N_{\text {used }}=\gamma_{r} Q_{r}+\gamma_{d} Q_{d}$. Note that $\left\{\mathcal{J}_{i, r}\right\}$ and $\left\{\mathcal{J}_{k, r}\right\}$ are disjoint for $i \neq k$. 
The proposed ranging signal design has $N_{c}=\gamma_{r} / 2$ frequency domain orthogonal codes (e.g., Walsh codes) of length $N_{c}$ each. Each code is repeated as $C_{i, r}\left(0, \ldots, \frac{\gamma_{r}}{2}-1\right)=$ $C_{i, r}\left(\frac{\gamma_{r}}{2}, \ldots, \gamma_{r}-1\right)$ to form a length- $\gamma_{r}$ ranging code. Each RSS transmits a ranging code randomly chosen from the above $N_{c}$ orthogonal codes on the chosen ranging subchannel defined by $\mathcal{J}_{i, r}$ only at one time slot randomly chosen from the $M$ ranging time slots. In our proposed scheme, each ranging time slot is equal to one OFDM symbol interval. The total number of ranging opportunities is $N_{\text {total }}=Q_{r} \cdot N_{c} \cdot M$.

Note that the channel responses within a ranging subchannel of the proposed scheme (e.g., $\gamma_{r}=8$ adjacent subcarriers in an OFDMA system with $N=2048$ subcarriers) are almost the same. Hence, the proposed ranging signals still maintain orthogonality at the BS (under the condition of negligible frequency offsets), while the ranging signals from $802.16 \mathrm{a} / \mathrm{e}$ suffer from loss of orthogonality due to the channel frequency selectivity.

\section{The Proposed Ranging Method}

We consider a TDD OFDMA system. Our proposed method first performs multi-user ranging signal detection, and then estimates timing offset for each detected RSS by utilizing the ranging signal detection results. Next, by combining the ranging signal detection results and the timing offsets estimation results, the received power of each detected RSS is estimated. The proposed ranging process is summarized in the following:

1) Each RSS estimates its channel power gains across the band by utilizing the pilots embedded in the downlink control frame. According to the estimated channel power gains, the RSS picks the ranging subchannel with the largest channel power gain, and adjusts the transmission power so that the received ranging signal power approximately equals to the target received ranging signal power $P_{r}$. If the adjusted transmission power is greater than the maximum allowable transmission power $P_{\max }$, then the RSS will not initiate the ranging process.

2) Each RSS randomly chooses one of the $N_{c}$ orthogonal codes and one of the $M$ ranging time slots, and then it transmits the ranging signal over the selected ranging subchannel in the selected ranging time slot.

3) At the BS, the receiver identifies whether or not a ranging code is transmitted without any collision, and extracts the timing and power information based on the detection results. For each detected active RSS, the BS compares estimated values of the timing offset and power with the ranging requirements. If they satisfy the requirements, then the BS informs the RSS that its ranging process is successful. If not, the BS sends timing and power adjustment parameters and a "continue" status notification to the RSS. The ranging response message from the BS also contains the code, ranging subchannel, and time slot where the corresponding RSS is detected. The BS need not send the collision status notification to the collided users.

4) When the RSSs receive the ranging response message, the RSSs with "success" status complete the ranging process, each of the RSSs with "continue" status will adjust the timing and power accordingly and randomly chooses a ranging code to continue the ranging process in the same ranging subchannel and time slot as the last ranging trial. The RSSs which do not find their ranging information in the ranging response message (i.e., the RSSs with collision) will each randomly choose a ranging code and a ranging time slot but use the same ranging subchannel to re-initiate the ranging process in the next frame or according to the truncated exponential backoff scheme.

When an RSS cannot detect the downlink control channel, or the ranging transmit power needed is larger than the maximum transmit power limit, the situation can be reflected in the received signal strength indicator at the RSS. This will alert the user to move around to get good channel to be able to start its ranging process.

\section{A. Multi-User Ranging Signal Detection}

At the BS after the $\mathrm{CP}$ removal and the $N$-point FFT operation, the frequency domain signal at the $k$ th subcarrier and the $m$ th symbol interval can be expressed as

$Y^{(m)}(k)=\sum_{i=1}^{N_{r}} X_{i, r}^{(m)}(k) H_{i, r}(k)+\sum_{j=1}^{N_{d}} X_{j, d}^{(m)}(k) H_{j, d}(k)+W^{(m)}(k)$

where

$$
\begin{gathered}
H_{i, \star}(k)=e^{\frac{j 2 \pi k d_{i, \star}}{N}} \sum_{l=0}^{L-1} h_{i, \star}(l) e^{\frac{-j 2 \pi l k}{N}}=e^{\frac{j 2 \pi k d_{i, \star}}{N}} \widetilde{H}_{i, \star}(k) \\
W^{(m)}(k)=\frac{1}{\sqrt{N}} \sum_{l=0}^{N-1} \omega\left(m\left(N+N_{g}\right)+N_{g}+l\right) e^{\frac{-j 2 \pi l k}{N}}
\end{gathered}
$$

At the $m$ th ranging time slot, the $\mathrm{BS}$ correlates the received frequency domain symbols of every $i$ th ranging subchannel with each $j$ th possible ranging code as

$$
\begin{aligned}
D 1_{i, j} & =\sum_{l=0}^{\frac{\gamma_{r}}{2}-1} Y^{(m)}\left(\mathcal{J}_{i, r}(l)\right) C_{j, r}^{*}(l) \\
D 2_{i, j} & =\sum_{l=\frac{\gamma_{r}}{2}}^{\gamma_{r}-1} Y^{(m)}\left(\mathcal{J}_{i, r}(l)\right) C_{j, r}^{*}\left(l-\frac{\gamma_{r}}{2}\right) .
\end{aligned}
$$

For simplicity, we drop the ranging code index $j$. Then the decision variable $D_{i}$ for the RSS detection at the $i$ th ranging subchannel is defined as

$$
D_{i}=\left|D 1_{i}\right|^{2}+\left|D 2_{i}\right|^{2}
$$

where the ranging time slot index $m$ is omitted for simplicity.

In the development of our RSS detector, we consider only 0 , 1 or 2 RSSs for each ranging opportunity, since the probability of 3 or more RSSs choosing the same ranging opportunity is small. Note that each RSS adjusts its transmission power to compensate for the power loss due to channel and hence $\left|A_{i} H_{i, r}\left(\mathcal{J}_{i, r}(l)\right)\right|^{2} \cong P_{r}$ for all $i$. The probability density function (PDF) of the $D_{i}$ is given by (14), where $s_{1}^{2}=\left|\sum_{l=0}^{\frac{\gamma_{r}}{2}-1} A_{i} H_{i, r}\left(\mathcal{J}_{i, r}(l)\right)\right|^{2}+\left|\sum_{l=\frac{\gamma_{r}}{2}}^{\gamma_{r}-1} A_{i} H_{i, r}\left(\mathcal{J}_{i, r}(l)\right)\right|^{2}$ $\cong 2\left(\frac{\gamma_{r}}{2}\right)^{2}\left|A_{i} H_{i, r}\left(\mathcal{J}_{i, r}(l)\right)\right|^{2}, s_{2}^{2}=4 \cos ^{2}\left(\frac{\theta_{1}-\theta_{2}}{2}\right) s_{1}^{2}$, and $\sigma^{2}$ $=\frac{\gamma_{r}}{4} \sigma_{w}^{2}$. Then the number of RSSs $n_{\mathrm{RSS}}$ within the same ranging opportunity is detected as

$$
n_{\mathrm{RSS}}=\underset{n=0,1,2}{\operatorname{argmax}}\left\{P_{n} \cdot f_{D_{i}}\left(D_{i} \mid n \mathrm{RSSs}\right)\right\}
$$


where $\left\{P_{n}\right\}$ representing the probability of $n$ RSSs in the same ranging opportunity can be replaced with pre-defined design values. This detection is equivalent to

$$
n_{\mathrm{RSS}}= \begin{cases}0, & \text { if } D_{i}<\eta_{0} \\ 1, & \text { if } \eta_{1}<D_{i}<\eta_{2} \\ 2, & \text { if }\left(\eta_{0} \leq D_{i} \leq \eta_{1}\right) \text { or }\left(D_{i} \geq \eta_{2}\right)\end{cases}
$$

where $\left\{\eta_{i}\right\}$ are the cross over points of $\left\{P_{n}\right.$. $\left.f_{D_{i}}\left(D_{i} \mid n \mathrm{RSSs}\right)\right\}$. In fact, we just need to check for $n_{\mathrm{RSS}}=1$ case which provides detected ranging codes and associated ranging subchannels and time-slots.

\section{B. Timing Offset Estimation}

For the collision-free $i$ th RSS, $D 1_{i}$ and $D 2_{i}$ can be approximated by

$$
\begin{aligned}
& D 1_{i} \cong A 1\left(\sum_{l=0}^{\frac{\gamma_{r}}{2}-1} e^{\frac{j 2 \pi \varepsilon_{i} \mathcal{J}_{i, r}(l)}{N}}\right)+\widetilde{W}_{1, i} \\
& D 2_{i} \cong A 2 e^{\frac{j 2 \pi \varepsilon_{i} \frac{\gamma_{r}}{2}}{N}}\left(\sum_{l=0}^{\frac{\gamma_{r}}{2}-1} e^{\frac{j 2 \pi \varepsilon_{i} \mathcal{J}_{i, r}(l)}{N}}\right)+\widetilde{W}_{2, i}
\end{aligned}
$$

where $A 1 \cong A 2=A_{i} \widetilde{H}_{i, r}\left(\mathcal{J}_{i, r}(l)\right), \varepsilon_{i}$ is the timing offset of the $i$ th RSS, and $\left\{\widetilde{W}_{1, i}, \widetilde{W}_{2_{i}}\right\}$ are i.i.d. zero-mean complex Gaussian random variables with a variance of $\sigma_{w}^{2} \gamma_{r} / 2$. Since $A 1 \cong A 2$, the timing offset can be estimated as

$$
\hat{\epsilon}_{i}=\operatorname{round}\left\{\frac{N}{\pi \gamma_{r}} \cdot \operatorname{angle}\left\{\frac{D 2_{i}}{D 1_{i}}\right\}\right\} .
$$

The above timing estimator provides a significant complexity reduction over the existing methods [3] [4] [5].

\section{RSS Power Estimation}

If only one ranging code is detected in one ranging slot, define

$$
\begin{aligned}
P 1_{i} & =\frac{2}{\gamma_{r}} \sum_{l=0}^{\frac{\gamma_{r}}{2}-1} Y^{(m)}\left(\mathcal{J}_{i, r}(l)\right) C_{j, r}^{*}(l) e^{\frac{-j 2 \pi \hat{\varepsilon}_{i} \mathcal{J}_{i, r}(l)}{N}} \\
P 2_{i} & =\frac{2}{\gamma_{r}} \sum_{l=\frac{\gamma_{r}}{2}}^{\gamma_{r}-1} Y^{(m)}\left(\mathcal{J}_{i, r}(l)\right) C_{j, r}^{*}\left(l-\frac{\gamma_{r}}{2}\right) e^{\frac{-j 2 \pi \hat{\varepsilon}_{i} \mathcal{J}_{i, r}(l)}{N}}(19)
\end{aligned}
$$

If there are more than one distinct ranging codes detected in one ranging slot, define

$$
P 1_{i}=\frac{2}{\gamma_{r}} D 1_{i}, \quad P 2_{i}=\frac{2}{\gamma_{r}} D 2_{i} .
$$

Then the power estimator is given by

$$
\hat{P}=\frac{\left(\left|P 1_{i}\right|^{2}-\frac{\sigma_{w}^{2}}{\frac{\gamma r}{2}}\right)+\left(\left|P 2_{i}\right|^{2}-\frac{\sigma_{w}^{2}}{\frac{\gamma r}{2}}\right)}{2}
$$

where the noise variance $\sigma_{w}^{2}$ is assumed to be known (can be measured or estimated easily).

\section{Average Ranging Transmission Power}

In our proposed ranging method, the system has $Q_{r}$ ranging subchannels and each ranging subchannel is composed of $\gamma_{r}$ adjacent subcarriers. For analytical tractability, in this section we consider a multipath Rayleigh fading channel with $Q_{r}$ sample-spaced i.i.d. taps (having a uniform power delay profile). The proposed equi-spaced ranging subchannels can be approximately modeled as $Q_{r}$ cyclically equi-spaced ranging subchannels. Let $H_{i, k}$ denote the channel gain of the $i$ th RSS $\left(i=0,1, \ldots, N_{R}-1\right)$ on the $k$ th $(k=0,1, \ldots, N-1)$ subcarrier. $\left\{H_{i, k}\right\}$ are circularly-symmetric complex Gaussian random variables with zero mean and unit variance. Then the average channel power gain of the $n$-th ranging subchannel for the $i$ th RSS is given by

$$
G_{i, n}=\frac{\sum_{k=\gamma_{r} \cdot n}^{\gamma_{r} \cdot(n+1)-1}\left|H_{i, k}\right|^{2}}{\gamma_{r}} \cong\left|H_{i, \gamma_{r} \cdot n}\right|^{2}, n=0,1, \ldots, Q_{r}-1 .
$$

From (22), we find that $\left\{G_{i, n}, n=0,1, \ldots, Q_{r}-1\right\}$ are approximately i.i.d. exponentially-distributed random variables with unit mean. The cumulative distribution function (CDF) of $G_{i, n}$ is given by

$$
F_{G}(g)=\left(1-e^{-g}\right) u(g)
$$

where $u(g)$ is the unit step function. In the proposed ranging method, the RSS picks the best channels based on its channel power gain $G_{i, n}$ defined in (22). Define

$$
Z_{i}=\max \left\{G_{i, n}: n=0,1, \ldots, Q_{r}-1\right\} .
$$

Then the CDF of $Z_{i}$ is

$$
F_{Z_{i}}(z)=\left(1-e^{-z}\right)^{Q_{r}} .
$$

Each RSS adjusts its transmission power such that

$$
P_{t, i} Z_{i}=P_{r}
$$

where $P_{t, i}$ is the adjusted transmission power of the $i$ th RSS and the $P_{r}$ is the target received power of an RSS at the BS. From (25) and (26), we obtain the PDF of $P_{t, i}$ as

$$
f_{P_{t, i}}(p)=\frac{P_{r}}{p^{2}} Q_{r}\left(1-e^{-\frac{P_{r}}{p}}\right)^{Q_{r}-1} e^{-\frac{P_{r}}{p}} .
$$

Then we can calculate the mean of the ranging transmission power as

$$
E\left[P_{t, i}\right]=P_{r} Q_{r} \sum_{n=0}^{Q_{r}-1}\left(\begin{array}{c}
Q_{r}-1 \\
n
\end{array}\right)(-1)^{n} E_{1}\left(\frac{P_{r} \cdot(n+1)}{P_{\max }}\right)
$$

where $E_{1}$ is the exponential integral defined by $E_{1}(x)=$ $\int_{x}^{\infty} e^{-t} t^{-1} d t$.

$$
f_{D_{i}}(x)= \begin{cases}\frac{1}{4 \sigma^{4} \Gamma(2)} x e^{-x / 2 \sigma^{2}}, & 0 \text { RSS } \\ \frac{1}{2 \sigma^{2}}\left(\frac{x}{s_{1}^{2}}\right)^{1 / 2} e^{-\left(s_{1}^{2}+x\right) / 2 \sigma^{2}} I_{1}\left(\frac{\sqrt{x} s_{1}}{\sigma^{2}}\right), & 1 \text { RSS } \\ \frac{1}{4 \pi^{2}} \int_{0}^{2 \pi} \int_{0}^{2 \pi} \frac{1}{2 \sigma^{2}}\left(\frac{x}{s_{2}^{2}}\right)^{1 / 2} e^{-\left(s_{2}^{2}+x\right) / 2 \sigma^{2}} I_{1}\left(\frac{\sqrt{x} s_{2}}{\sigma^{2}}\right) d \theta_{1} d \theta_{2}, & 2 \text { RSSs }\end{cases}
$$



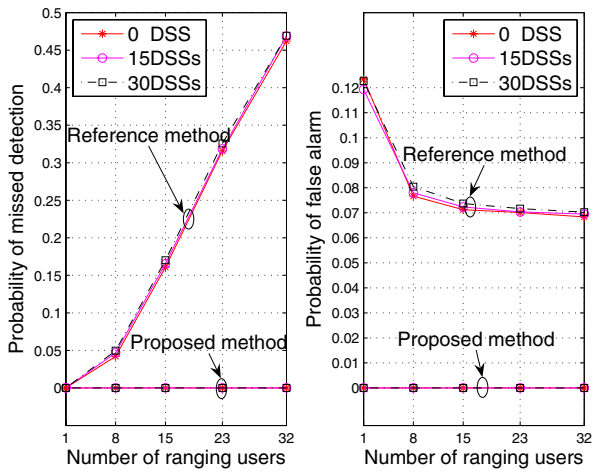

Fig. 1. The probabilities of missed detection and false alarm at the first ranging frame

\section{Simulation Results AND Discussion}

\section{A. Simulation Parameters}

For comparison, the ranging code detector and timing estimator in [3] with the ranging signals from the IEEE 802.16e are used as the reference method. The system parameters, selected from [2], are summarized as follows. The uplink bandwidth is $3 \mathrm{MHz}$, the sub-carrier spacing is $1.64 \mathrm{kHz}$, and $N=2048$ sub-carriers. The DC sub-carriers is not used. The numbers of left and right null sub-carriers are 184 and 183, respectively. We use BPSK for RSS and QPSK for DSS. The number of data sub-carriers per sub-channel, $\gamma_{d}$, is 48 . The number of sample-spaced channel taps, $L$, is set to 16 . Channels of different users are assumed to be independent. We consider a cell radius of $4.5 \mathrm{~km}$ which gives the maximum transmission delay (round trip) $d_{\max , \mathrm{r}}=104$ samples. $N_{g}$ is set to 128 samples satisfying the condition $d_{\text {max }, \mathrm{r}}<N_{g}-L$. The number of OFDM symbols in one uplink frame is set to 6 . The timing requirement based on [2] is that all uplink OFDM symbols should arrive at the BS within an accuracy of $\pm 25 \%$ of the minimum guard-interval or better, so the timing offset should be within \pm 16 samples. We set $d_{\text {max,d }}=32$. The power requirement is that SNR of each RSS at the BS should be above $9.4 \mathrm{~dB}$. The system parameters are the same for the proposed ranging method and the reference ranging method. The difference is the ranging channel setup. For the proposed method, the ranging channel is composed of 16 ranging subchannels, and each ranging sub-channel has 8 contiguous subcarriers. The number of orthogonal codes (and the code length) is $N_{c}=4$. The total number of ranging opportunities in the proposed method is $N_{\text {total }}=16 \cdot 4 \cdot 6=384$.

For the reference method, each RSS transmits one selected CDMA code twice on a single ranging channel that consists of 144 non-contiguous subcarriers over two consecutive symbols. The number of ranging codes is 256 . The code set is composed of three categories: initial ranging codes, periodic ranging codes and bandwidth requests codes. If we assume that 128 codes are assigned for the initial ranging, then the total number of ranging opportunities is $N_{\text {total }}=128 \cdot \frac{6}{2}=384$ which is the same as that in the proposed method. In order to reduce the simulation time for the reference method, we use only 32
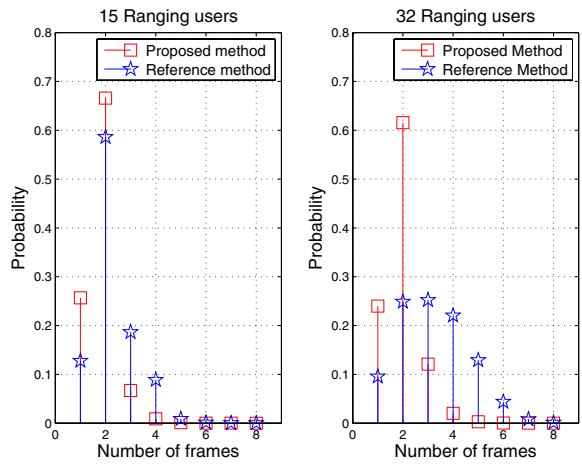

Fig. 2. The probability distribution of the number of frames required for a successful ranging

codes which is equal to the considered maximum number of RSSs and we assume there is no collision in the reference method. Since there is no power estimator provided in [3], we assume perfect power estimation for the reference method. Hence the performance of the reference method presented will be slightly optimistic. For simplicity in evaluation, we assume that all $N_{r}$ RSSs attempt their ranging simultaneously at the first ranging frame, there are no new RSSs until their ranging processes are completed and collided RSSs retry their ranging in the next frame. The frequency offsets (normalized by the subcarrier spacing) of the RSSs are modeled as i.i.d. uniform random variables over the range of $[-0.02,0.02]$.

For the reference method, the initial ranging transmission power on each ranging subcarrier is set $10 \mathrm{~dB}$ above the noise power $\left(\sigma_{w}^{2}\right)$ on one subcarrier. So the total ranging transmission power over 144 ranging subcarriers normalized by $\sigma_{w}^{2}$ is $10 \log _{10}(1440) \mathrm{dB}$. In the proposed method, the target received total ranging signal power of an RSS is set the same as the statistical average of the total received ranging signal power of an RSS in the reference method, i.e., $P_{r} / \sigma_{w}^{2}=1440 / 8$. Under this setting, the reference method utilizes twice the ranging transmission energy of the proposed method without power control and multiuser diversity since the former transmits on two OFDM symbols while the latter sends on one OFDM symbol interval.

\section{B. Simulation Results and Discussions}

Fig. 1 shows that probabilities of missed detection and false alarm evaluated under collision-free condition. The probability of missed detection $P_{\text {miss }}$ is defined as $E\left[\frac{D_{m}}{N_{r}}\right]$ where $D_{m}$ is the number of RSSs whose transmitted ranging signals are not detected at the BS and $N_{r}$ is the total number of RSSs. The probability of false alarm $P_{\text {false }}$ is defined as $E\left[\frac{D_{a}}{N_{\text {total }}-N_{r}}\right]$ where $D_{a}$ is the number of ranging signals which are detected at the BS but are not transmitted from any RSSs. Neither missed detection nor false alarm are observed for the proposed method. The $P_{\text {miss }}$ and $P_{\text {false }}$ performances of the proposed method are robust to the DSS interference and RSS interference, while the reference method's performances substantially degrade as the number of the RSSs increases.

Fig. 2 shows the probability distribution of the number of frames required for an RSS to complete a successful 

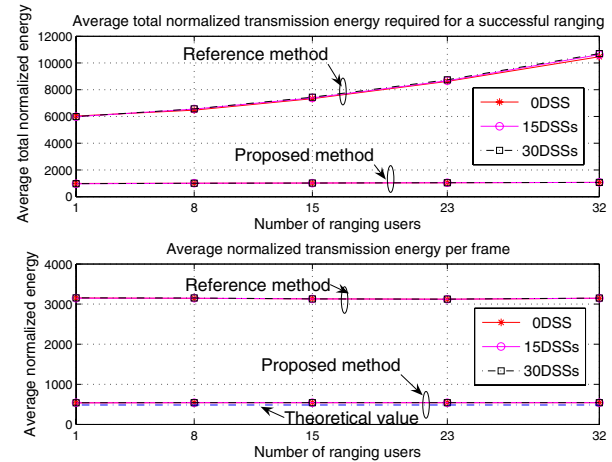

Fig. 3. Average normalized ranging transmission energy comparison

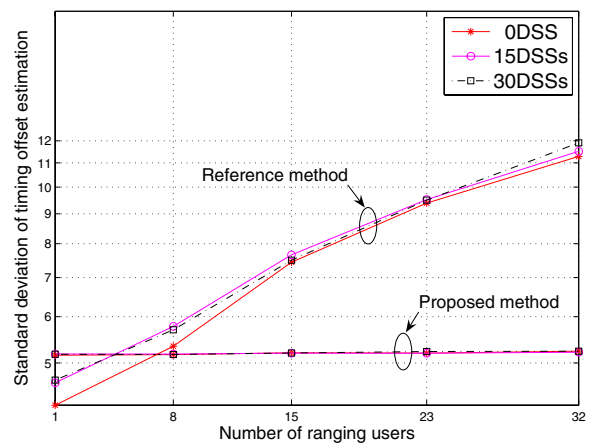

Fig. 4. Timing offset estimation performance comparison

ranging. For 15 RSSs case, the simulation results show that about $90 \%$ of the RSSs in the proposed method ( $70 \%$ in the reference method) can finish the ranging process in two frames. For 32 RSSs case, we can see that the proposed method yields a greater advantage $(85 \%$ versus $35 \%$, i.e., over $200 \%$ improvement) over the reference method. This advantage translates into smaller latency at the network entry.

Fig. 3 compares the ranging transmission energy normalized by the noise energy contained on one subcarrier in one OFDM symbol duration. The upper subplot shows the average of total normalized ranging transmission energy required for an RSS to finish its ranging process. The lower subplot shows the average normalized ranging transmission energy per ranging frame for an RSS, and we also include the theoretical result of the average normalized transmission energy for the proposed method as given in (28). The proposed method cuts down the ranging energy consumption at an RSS to approximately $\frac{1}{6}$ of that required in the reference method. The close match between simulation and theoretical results also validates our theoretical analysis in Section V.

Fig. 4 shows the standard deviation of the timing offset estimation versus the number of RSSs for 0,15 and 30 DSSs. The true timing offsets for RSSs and DSSs are taken randomly from the interval $\left[0, d_{\max , \mathrm{r}}\right]$ and $\left[0, d_{\max , \mathrm{d}}\right]$, respectively. The simulation results show that both methods are robust to the DSS interference. The proposed timing estimator is also robust to the RSSs interference, while the performance of the reference estimator degrades as the number of RSSs increases.

Fig. 5 shows the normalized power estimation MSE defined

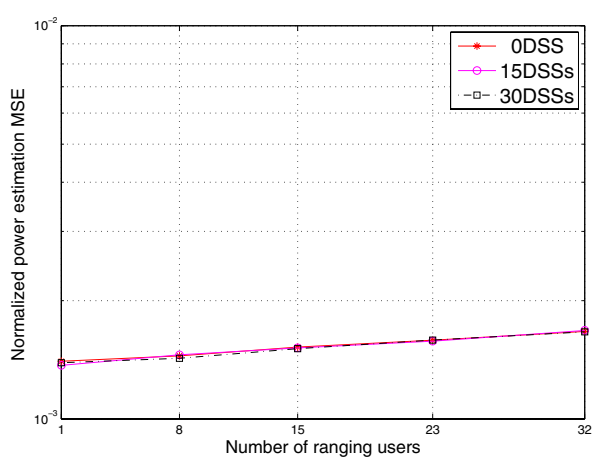

Fig. 5. Power estimation performance

as $E\left[\left(1-\frac{\hat{P}}{P}\right)^{2}\right]$ versus the number of RSSs for 0 DSS, 15 DSSs and 30 DSSs. Since there is no power estimator provided in [3], we only show the performance of the proposed power estimator. We can see that the proposed power estimator has very good performance and is robust to the DSS interference.

The computational complexity for the ranging code detection and timing estimation in one ranging frame for the proposed method is $\frac{1}{4046}$ times the complexity of the reference method. Detailed complexity expressions are skipped due to space limitation.

The simulation results demonstrate that the proposed method substantially outperforms the reference method in all considered aspects. Note that we have assumed collisionfree condition and perfect power estimation for the reference method. Without this assumption, the proposed method will yield even larger gains than those illustrated in this paper.

\section{CONCLUSIONS}

We have presented a novel initial ranging method that takes advantage of multiuser diversity in OFDMA uplink. We have developed a new ranging signal design which provides multiuser diversity gain and facilitates new efficient lowcomplexity algorithms for multiuser ranging signal detection, timing offset estimation and power estimation. The proposed ranging method achieves significant gains in ranging signal detection and synchronization performance, tremendous energy saving at the subscriber stations, and huge complexity reduction over the existing ranging methods while utilizing the same amount of time and frequency resources.

\section{REFERENCES}

[1] Broadband Wireless Access: IEEE MAN standard, IEEE LAN/MAN Standards Committee IEEE 802.16a, 2003.

[2] Broadband Wireless Access: IEEE MAN standard, IEEE LAN/MAN Standards Committee IEEE 802.16e, 2005.

[3] J. Krinock, M. Singh, M. Paff, Vincent, A. Lonkar, L. Fung, and C.C. Lee, "Comments on OFDMA ranging scheme described in IEEE 802.16ab-01/01r1," Document IEEE 802.16abc-01/24.

[4] H. Mahmoud, H. Arslan, and M. Ozdemir, "Initial ranging for WiMAX (802.16e) OFDMA," in Proc. IEEE Military Communications Conference, Oct. 2006, pp. 1-7.

[5] X. Fu and H. Minn, "Initial uplink synchronization and power control (ranging process) for OFDMA systems," in Proc. IEEE GLOBECOM, vol. 6, Nov. 29-Dec. 3 2004, pp. 3999-4003.

[6] X. Fu, Y. Li, and H. Minn, "A new ranging method for OFDMA systems," IEEE Trans. Wireless Commun., vol. 6, no. 2, pp. 659-669, Feb. 2007. 